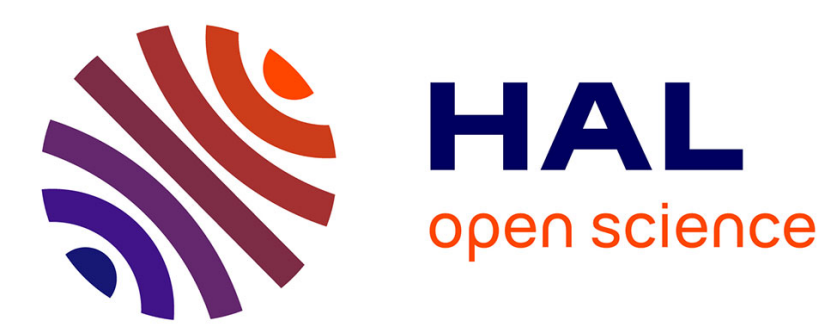

\title{
Subcritical transition to turbulence in plane Couette flow
}

F. Daviaud, J. Hegseth, P. Bergé

\section{To cite this version:}

F. Daviaud, J. Hegseth, P. Bergé. Subcritical transition to turbulence in plane Couette flow. Physical Review Letters, 1992, 69 (17), pp.2511 - 2514. 10.1103/PhysRevLett.69.2511 . cea-01374002

\section{HAL Id: cea-01374002 https://hal-cea.archives-ouvertes.fr/cea-01374002}

Submitted on 29 Sep 2016

HAL is a multi-disciplinary open access archive for the deposit and dissemination of scientific research documents, whether they are published or not. The documents may come from teaching and research institutions in France or abroad, or from public or private research centers.
L'archive ouverte pluridisciplinaire HAL, est destinée au dépôt et à la diffusion de documents scientifiques de niveau recherche, publiés ou non, émanant des établissements d'enseignement et de recherche français ou étrangers, des laboratoires publics ou privés. 


\title{
Subcritical Transition to Turbulence in Plane Couette Flow
}

\author{
F. Daviaud, J. Hegseth, and P. Bergé \\ Service de Physique de l'Etat Condensé, Centre d'Etudes de Saclay, F-91191 Gif-sur-Yvette CEDEX, France
}

(Received 21 May 1992)

\begin{abstract}
The transition to turbulence in plane Couette flow was studied experimentally. The subcritical aspect of this transition is revealed by the stable coexistence of laminar and turbulent domains. By perturbing the flow, a critical Reynolds number has been determined, above which an artificially triggered turbulent spot can persist. The study of the spatiotemporal evolution of these spots shows, among other things, the existence of waves traveling away from the turbulent regions.
\end{abstract}

PACS numbers: $47.20 .-\mathrm{k}, 47.15 .-\mathrm{x}$

Two types of transitions to turbulence can generally be distinguished. Those where disorder appears progressively, as a small perturbation of the regular state, and those for which the systems experience an abrupt and often localized transition from a regular state to a highly disordered one [1]. Following an analogy with bifurcation theory, the former can be called supercritical and the latter subcritical [2]. Many examples of the two types of transition to turbulence can be found in the literature. Circular Couette flow with the outer cylinder at rest or with the two cylinders corotating [3] is a good example of a system showing a supercritical transition, while counterrotating circular Couette flow [4] or plane Poiseuille flow [5] are known to exhibit turbulent spots coexisting with the otherwise laminar flow. This metastability of two states-one regular (laminar) and one irregular (turbulent) - is typical of a subcritical bifurcation. However, very few experiments involving plane Couette flow have been performed, though it appears to be one of the simplest flows likely to display a subcritical transition to turbulence.

Plane Couette flow (PCF) corresponds to a linear velocity profile which results from the relative motion of two infinite parallel walls at a given distance apart. It is an exact solution of the governing nonlinear equations and it is believed to remain linearly stable at all $R$ [6] ( $R=U h / v$, where $U$ is the speed of either wall, $h=d / 2$ is half the gap, and $v$ is the kinematic viscosity). This theoretical result suggests that instability in PCF has a nonlinear origin and can be generated by finite-amplitude perturbations. Orszag and Kells have shown numerically [7] that only three-dimensional disturbances can drive a transition to turbulence at Reynolds numbers larger than $R \simeq 1000$ (with our definition of $R$ ) and Nagata [8] has discovered the existence of 3D finite-amplitude solutions of PCF for $R>125$, which provides a lower bound for instability and turbulence. Recently, Lerner and Knobloch [9] and Dubrulle and Zahn [10] have studied the influence of a small defect on the onset of instability for inviscid and viscous PCF, respectively. The development of turbulent spots in PCF was also studied by means of direct numerical simulations by Lundbladh and Johansson [11], and a critical Reynolds number $R \simeq 375$ was found, above which localized turbulent regions can persist.

Facing these theoretical and numerical works, only a few experimental studies of transition in PCF have been carried out, probably due to the experimental difficulties encountered when running and making measurements in a PCF apparatus. Reichardt [12] has observed turbulence for $R>750$ and Leutheusser and Chu [13] for $R>280$. More recently, Tillmark and Alfredsson have reported the existence of a transitional Reynolds number $R \simeq 360$, which is the lowest $R$ for which a transition to turbulence occurs if a strong enough disturbance is applied [14]. In this Letter, we report experimental results concerning the transition to turbulence in a PCF. By perturbing the flow, we have both determined a critical Reynolds number above which turbulence persists and characterized the transition. We have also studied the spatiotemporal evolution of the turbulent spots, which exhibits the existence of waves traveling away from the turbulent regions.

Several different geometries have been tried in attempts to make PCF [12-16]. Our system utilizes a geometry which results in no mean flow, i.e., the walls move in opposite directions with the same velocity [17]. In this geometry the two vertically oriented parallel walls move in a horizontal direction. We define these walls by using an endless transparent plastic film belt $(363.0 \mathrm{~cm}$ long, $25.4 \mathrm{~cm}$ wide, and $0.16 \mathrm{~mm}$ thick), two pairs of small rotating Plexiglas cylinders, and two large rotating Plexiglas cylinders as shown schematically in Fig. 1. The endless belt is driven, through friction, by one of the two large cylinders $(12.00 \mathrm{~cm}$ in diameter $)$ which are placed at opposite ends of the system. The cylinder which drives the belt is mechanically coupled to an ac motor which drives the system. The two "infinite" parallel planes are defined by guiding the endless belt through two pairs of smaller cylinders $7.0 \mathrm{~mm}$ apart, all of which are parallel to the large cylinders as shown in Fig. 1. The belt is also guided by two glass plates, one of which is black and the other transparent, defining a test section of $1 \mathrm{~m}$. Using $h=d / 2$ as a length scale, the transverse aspect ratio $\Gamma_{z}$ (dimensionless width of the channel) is $\Gamma_{z} \approx 70$ and the longitudinal aspect ratio is (dimensionless length of the 


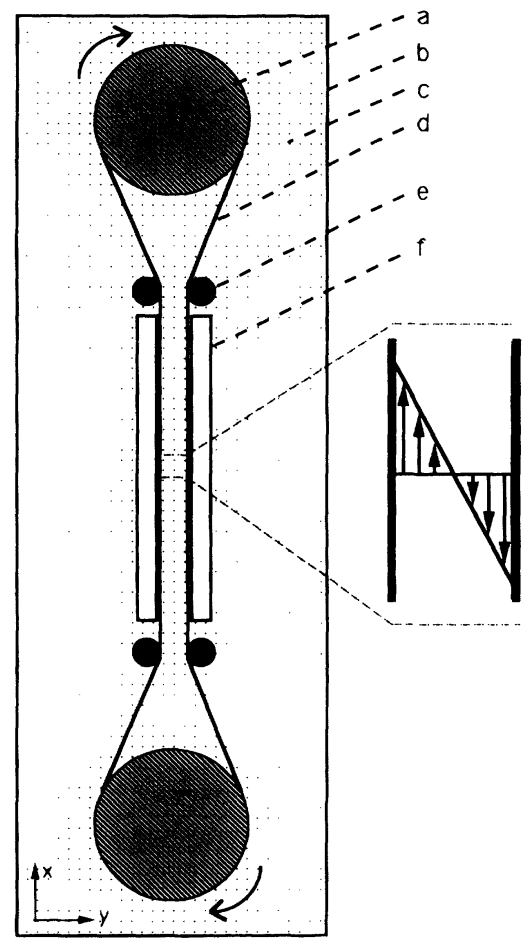

FIG. 1. Schematic drawing of the experimental apparatus in a horizontal plane. (a) Large cylinders, (b) tank, (c) water, (d) clear plastic belt, (e) small cylinders, and (f) glass plates.

channel) $\Gamma_{x} \approx 285$. We have chosen to use a transparent belt so that optical methods in data acquisition, such as laser Doppler velocimetry (LDV) and flow visualization with image processing, may be used. The entire assembly is placed in a glass tank which is filled with water, our working fluid, which has a viscosity of $v \simeq 0.01$ stoke at $293 \mathrm{~K}$.

We visualize the laminar and turbulent regions by seeding the flow with a dilute solution of Merck Iriodin 100 Silver Pearl which consists of thin and flat reflective mica platelets $(0.1 \mu \mathrm{m}$ thick and $10 \mu \mathrm{m}$ across $)$ which align, on average, with the stream planes of the flow. They also respond very quickly to any local change in the flow field, giving an almost instantaneous change in the light reflectance field whenever there is a change in the velocity field. This makes Iriodin an excellent indicator of turbulence on all scales [18]. When the flow is seeded with Iriodin, turbulence is indicated by a relatively rapid fluctuation in the reflected light field whereas in the laminar flow the light reflectance is steady. The light reflectance field may then be recorded using a video camera and subsequently digitized and analyzed by image processing. We have also visualized the velocity field in two dimensions by seeding the flow with a white ceramic powder (Pyroceram 7575, 100 Mesh) and illuminating the flow with an argon laser light sheet in a vertical or horizontal plane. The light scattered by the particles is reflected by a mirror into a camera for recording. In this manner, we have checked that the velocity profile is linear for $R$ up to 300 by following the particles in the streamwise cross section [17].

Artificially triggered disturbances are made by a turbulent jet which is injected into the laminar flow from a hole machined in the dark glass plate. This injection is controlled by an electrovalve that is activated when the 2-mm hole in the plastic belt is aligned with the hole of the plate. The amplitude of the disturbance can also be controlled by adjusting the pressure of the jet.

The system has first been studied without being artificially disturbed, in order to study the intrinsic noise of the experiment. For $R<400$ the turbulence is localized at the entrances of the test section and does not invade the laminar flow during observation times of more than $30 \mathrm{~min}$. For $R \geq 400$, turbulence invades the system under the form of two fronts coming from each side of the test section and contaminating the laminar flow. The size of the part that is contaminated by turbulence increases with $R$ while the time of invasion decreases with $R$. For $R=450$ and after $5 \mathrm{~min}$, all of the test section has been invaded [19].

Turbulent spots were initiated at given values of $R$, as described above, and their evolution was recorded in the $(x, z)$ plane ( $x$ and $z$ refer to the streamwise and spanwise directions, respectively). As expected, they are not advected with a mean velocity, but remain in the test section of the experiment. Moreover, as they are created in the center of the belt, they appear as quasisymmetrical ellipsoids, their shape being stretched in the streamwise direction (cf. Fig. 2).

We have studied the evolution of the spots with $R$ and determined the minimum $R_{c}$ for the subcritical transition to turbulence. For $R<R_{c}=370 \pm 10$, the turbulent spots generated in the laminar flow relax in short times [cf. Fig. 2(a)], while for $R \geq R_{c}$, they are self-sustaining for arbitrarily long times [cf. Fig. 2(b)]. The mean relaxation time $\tau$ of the spots was measured by repeating the procedure a large number of times for each value of $R$. The evolution of $\tau$ with $R$ exhibits a divergence of $\tau$ near $R_{c}$ (cf. Fig. 3). Moreover, the fluctuations of the relaxation times around the mean value increase when approaching $R_{c}$. The transition thus appears to have some characteristics of critical phenomena [20] and work is in progress to characterize it quantitatively [19].

The influence of the amplitude of the perturbation on the transition has been studied by adjusting the velocity of the turbulent jet that creates the perturbation. This amplitude does not seem to significantly affect the threshold $R_{c}$, but it does, however, influence the aspect of the transition: The mean relaxation time increases with the amplitude of the perturbation, especially near $R_{C}$ [19].

When $R>R_{c}$, the turbulent spots first spread very quickly (spreading rate $\sim 0.3 h \mathrm{sec}^{-1}$ ) and saturate to a macroscopic size which depends on the value of $R$. Then, they have a slow spatial and temporal evolution, but occupy the same mean space. Their position and shape 

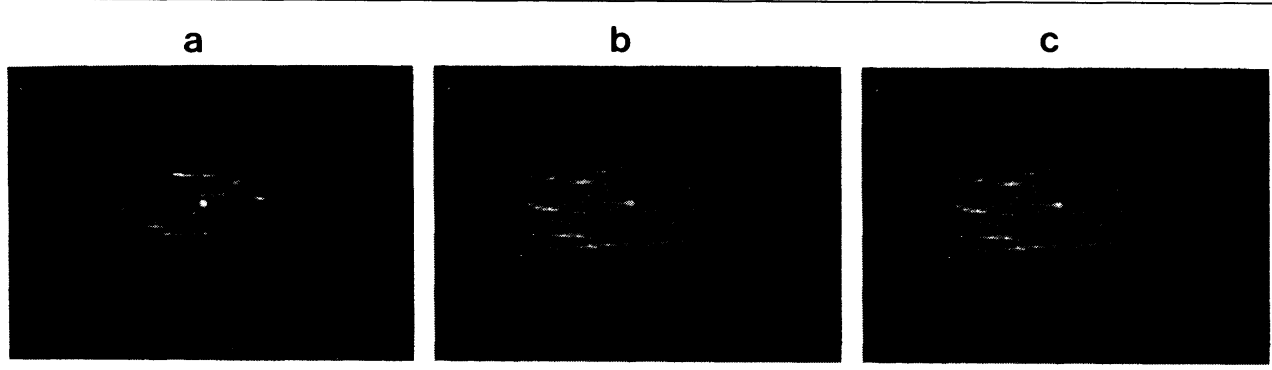

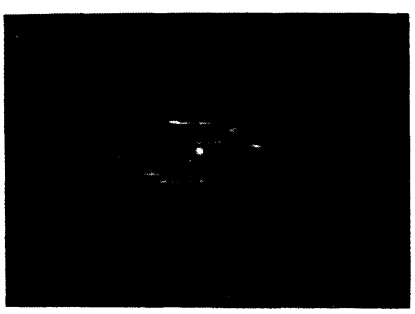

d

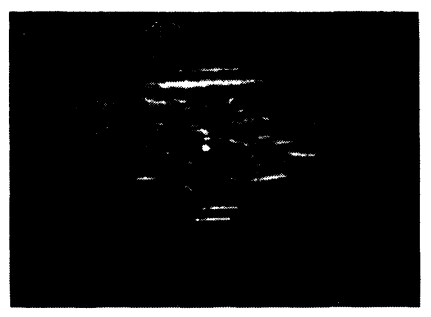

e

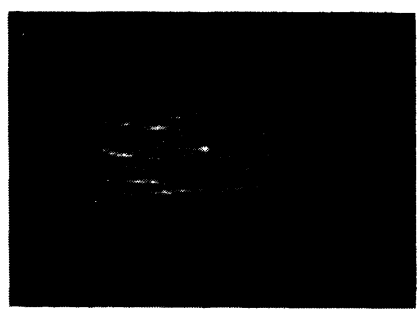

f

FIG. 2. Evolution of a turbulent spot after a perturbation; the time interval between photographs is $5 \mathrm{sec}$. (a),(b),(c) $R=350$; (d),(e),(f) $R=380$.

evolve more slowly than the time for fluctuations of light reflectance within the turbulent regions. Successive images have been digitized in the $(x, z)$ plane and a rms calculation shows that the maximum light fluctuations correspond to the turbulent spot. We have also observed vortical structures in the spanwise $(y, z)$ plane [19].

Two types of regions (laminar and turbulent) can easily be distinguished from an experimental viewpoint. Turbulence is seen to invade the laminar flow by a process of contamination of the regions adjacent to the spot. The edges of the turbulent patches are usually either curved or straight and inclined to the horizontal. Throughout the turbulent region horizontal streaks can be seen, and these streaks propagate outward from the spot in the vertical (spanwise) direction. The spatiotemporal evolu-

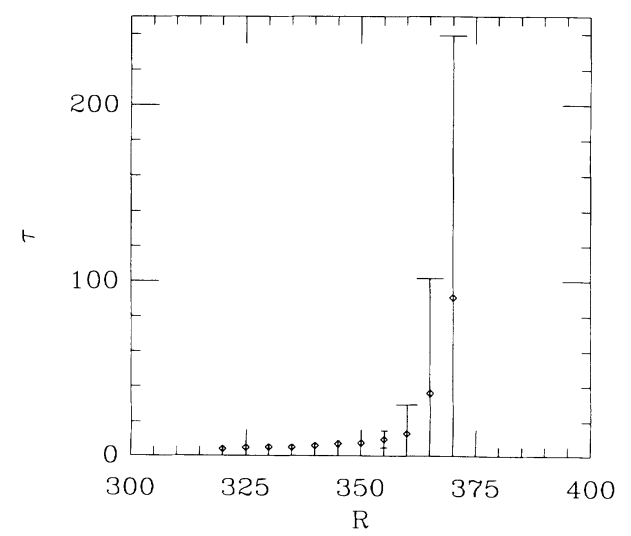

FIG. 3. Evolution of the relaxation time $\tau$ of the artificially triggered turbulent spots as a function of the Reynolds number $R$. tion of a spot and the existence of traveling waves are displayed in the diagram of Fig. 4. This diagram is obtained by digitizing the image along a vertical line $(z)$ situated in the middle of the test section, and plotting the evolution of the intensity along this line as a function of time. The wavelength of these waves is $\sim 2 h$ in the streamwise direction and their phase velocity is $\sim 2 h$ $\sec ^{-1}$ near $R_{c}$. They could play an important role in the propagation of turbulence, particularly in the spanwise direction.

As $R$ is further increased, the spots can move, grow, split into two spots, or decay and a regime reminiscent of spatiotemporal intermittency is observed [21]. This regime will be characterized elsewhere, but the mean fraction of total space which is turbulent can be defined and it appears to increase with $R$ (at $R \simeq 450$, nearly all the test section is turbulent). Near $R=400$, the turbulence

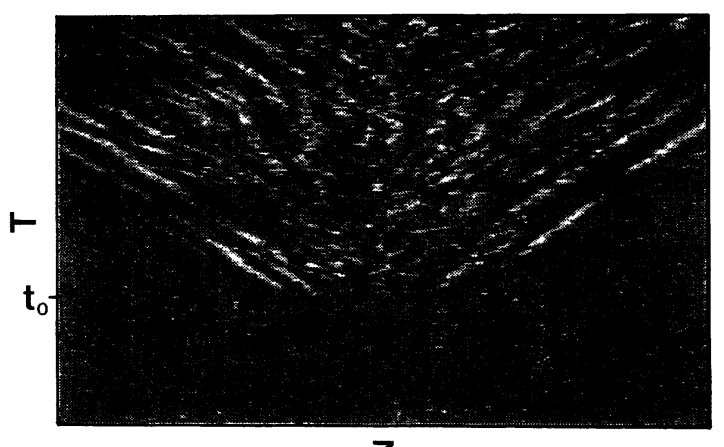

$\mathbf{Z}$

FIG. 4. Spatiotemporal evolution of a turbulent spot initiated at $t_{0}$. Spatial digitization is made over 512 pixels and the total observation time is $25 \mathrm{sec}$. 
may take a form that is similar to the spots that are observed in plane Poiseuille flow [5] or in the Blasius boundary layer [22]. They can also form straight inclined patches across the $(x, z)$ plane, a shape similar to that of the spiral turbulence observed in circular Couette flow [4].

A PCF apparatus has been constructed in which we have observed the subcritical transition to turbulence at $R=R_{c}$, by perturbing the flow. Below $R_{c}$, the laminar state is stable to finite-amplitude perturbations, and above $R_{c}$, the perturbations do not relax and a regime of intermittent turbulence is observed. Although many aspects are still to be investigated, the transition appears to be a critical phenomenon. After submitting this paper, we became aware of similar results obtained by Tillmark and Alfredsson [23]. By perturbing a PCF by different means (air bubble situated at the bottom), they have found a value of $R_{c}$ very close to our measurements. They have also noticed the creation of waves at the wing tips of the spots, which appear to be similar to those displayed in Fig. 4. Our experimental results are also in very good agreement with the numerical results obtained by Lundbladh and Johansson [11], especially for the critical Reynolds number $R_{c}$. However, the existence of waves was not predicted by their simulations nor was the intermittent regime which is observed above the threshold. Work is currently under progress in this direction.

We wish to thank B. Bonetti, G. Balzer, P. Manneville, and Y. Pomeau for stimulating discussions and M. Labouise, P. Hede, and B. Ozenda for their technical assistance.

[1] See, e.g., D. J. Tritton, Physical Fluid Dynamics (Van Nostrand Reinhold, New York, 1977).

[2] Y. Pomeau, Physica (Amsterdam) 23D, 1 (1986); 51D, 546 (1991).

[3] D. Coles, J. Fluid Mech. 21, 385 (1965). Also see C. W.
Van Atta, J. Fluid Mech. 25, 495 (1966).

[4] C. D. Andereck, S. S. Liu, and H. L. Swinney, J. Fluid Mech. 164, 155 (1986); J. J. Hegseth, C. D. Andereck, F. Hayot, and Y. Pomeau, Phys. Rev. Lett. 62, 257 (1989).

[5] D. R. Carlson, S. E. Widnall, and M. F. Peeters, J. Fluid Mech. 121, 487 (1982).

[6] See, e.g., P. G. Drazin and W. H. Reid, Hydrodynamic Stability (Cambridge Univ. Press, Cambridge, 1981).

[7] S. A. Orszag and L. Kells, J. Fluid Mech. 96, 159 (1980).

[8] M. Nagata, J. Fluid Mech. 217, 519 (1990).

[9] J. Lerner and E. Knobloch, J. Fluid Mech. 189, 117 (1988).

[10] B. Dubrulle and J. P. Zahn, J. Fluid Mech. 231, 561 (1991).

[11] A. Lundbladh and A. Johansson, J. Fluid Mech. 229, 499 (1991).

[12] H. Reichardt, Z. Angew. Math. Mech. Sonderheft 26, (1956).

[13] H. J. Leutheusser and V. H. Chu, J. Hydraul. Div. Am. Soc. Civ. Eng. 97, 1269 (1971).

[14] N. Tillmark and P. H. Alfredsson, Bull. Am. Phys. Soc. 35, 2248 (1990).

[15] J. M. Robertson and H. F. Johnson, J. Eng. Mech. Div. Am. Soc. Civ. Eng. 96, 1171 (1970).

[16] M. Aydin and J. Leutheusser, Rev. Sci. Instrum. 50, 1362 (1979).

[17] J. Hegseth, F. Daviaud, and P. Bergé, in Ordered and Turbulent Pattern in Taylor-Couette Flow, edited by C. D. Andereck (Plenum, New York, 1991).

[18] K. W. Schwarz, Phys. Rev. Lett. 64, 415 (1990).

[19] F. Daviaud and J. Hegseth (to be published).

[20] See, e.g., J. D. Gunton, M. San Miguel, and P. S. Sahni, in Phase Transitions and Critical Phenomena, edited by C. Domb and J. L. Lebowitz (Academic, New York, 1983), Vol. 8.

[21] F. Daviaud, M. Bonetti, and M. Dubois, Phys. Rev. A 42 , 3388 (1990).

[22] B. Cantwell, D. Coles, and P. Dimotakis, J. Fluid Mech. 87, 641 (1978).

[23] N. Tillmark and P. H. Alfredsson, J. Fluid Mech. 235, 89 (1992). 
a
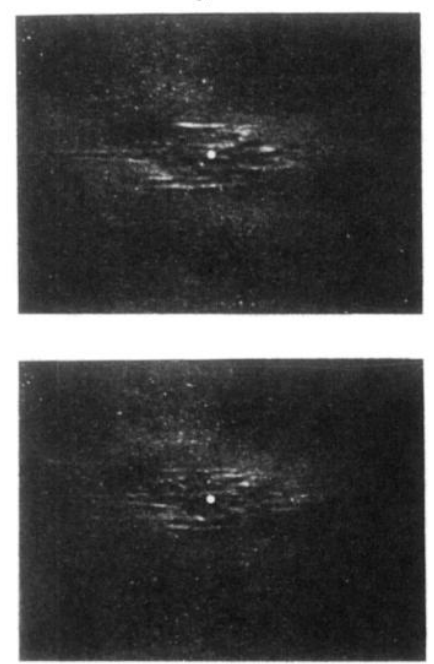

d b
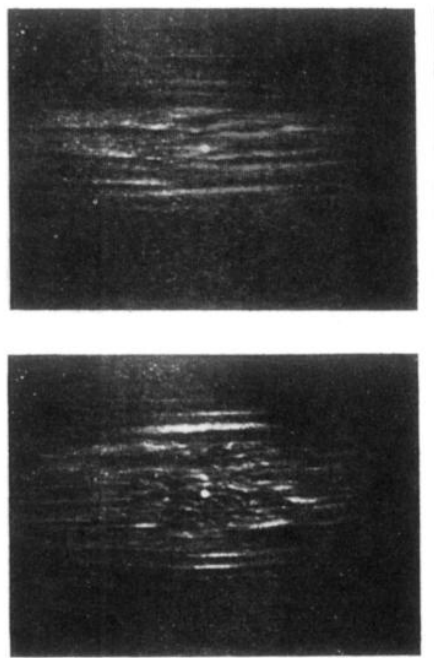

e
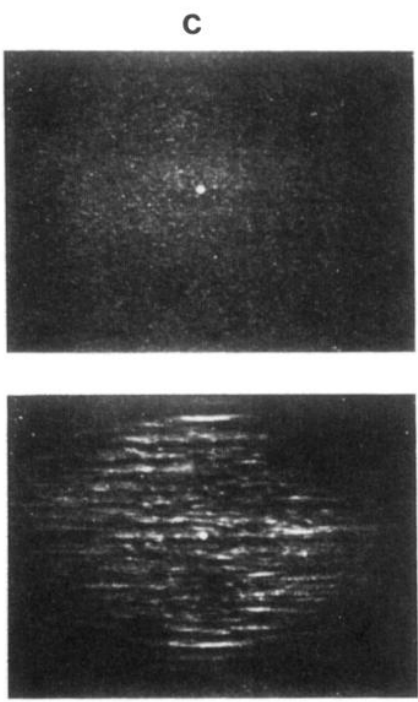

f

FIG. 2. Evolution of a turbulent spot after a perturbation; the time interval between photographs is 5 sec. (a),(b),(c) $R=350$; (d),(e),(f) $R=380$. 


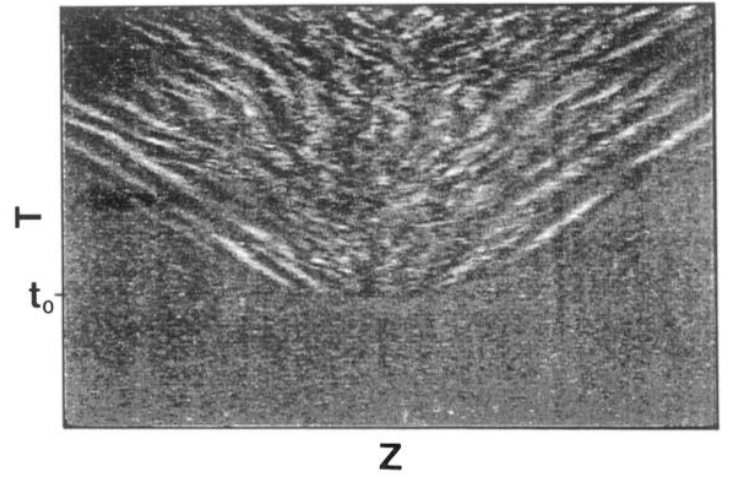

FIG. 4. Spatiotemporal evolution of a turbulent spot initiated at $t_{0}$. Spatial digitization is made over 512 pixels and the total observation time is $25 \mathrm{sec}$. 